\title{
Color Texture Analysis and Classification: An Agent Approach Based on Partially Self-avoiding Deterministic Walks
}

\author{
André Ricardo Backes ${ }^{1}$, Alexandre Souto Martinez ${ }^{2}$, \\ and Odemir Martinez Bruno ${ }^{3}$ \\ 1 Faculdade de Computação - Universidade Federal de Uberlândia \\ Uberlândia MG Brasil \\ backes@facom.ufu.br \\ 2 Faculdade de Filosofia, Ciências e Letras de Ribeirão Preto (FFCLRP) \\ Universidade de São Paulo (USP) \\ Av. Bandeirantes, 3900 \\ 14040-901 Ribeirão Preto, SP, Brazil \\ asmartinez@ffclrp.usp.br \\ 3 Instituto de Física de São Carlos (IFSC) \\ Universidade de São Paulo (USP) \\ Avenida do Trabalhador São-carlense, 400 \\ 13560-970 São Carlos SP Brazil \\ bruno@ifsc.usp.br
}

\begin{abstract}
Recently, we have proposed a novel approach of texture analysis that has overcome most of the state-of-art methods. This method considers independent walkers, with a given memory, leaving from each pixel of an image. Each walker moves to one of its neighboring pixels according to the difference of intensity between these pixels, avoiding returning to recent visited pixels. Each generated trajectory, after a transient time, ends in a cycle of pixels (attractor) from where the walker cannot escape. The transient time $(t)$ and cycle period $(p)$ form a joint probability distribution, which contains image pixel organization characteristics. Here, we have generalized the texture based on the deterministic partially self avoiding walk to analyze and classify colored textures. The proposed method is confronted with other methods, and we show that it overcomes them in color texture classification.
\end{abstract}

Keywords: partially self-avoiding deterministic walks, texture analysis, color images.

\section{Introduction}

Texture is one of the most important visual attribute in computer vision and image analysis. It is a visual pattern which, in digital images, consists of sub-patterns. The sub-patterns are related to the pixel distribution in an image region and its characteristics, such as size, brightness and color. Although, there is no exact definition for texture in the literature, it is an attribute naturally comprehended by

I. Bloch and R.M. Cesar, Jr. (Eds.): CIARP 2010, LNCS 6419, pp. 6-13, 2010.

(C) Springer-Verlag Berlin Heidelberg 2010 
humans and responsible to improve the human vision process. Texture importance is presented in Ref. [2] for both, natural and artificial vision. In computer vision, there is a huge number of texture applications that are found in various areas. These applications range from aiding diagnoses in medical images [3], analysis of geological structures in images [4], microscope images [5] etc.

Texture has been thoroughly studied and many methods have been proposed to solve and improve the analysis 26 . These methods can be grouped in: image pixels spectral analysis (e.g., Fourier descriptors [7] and Gabor filters [8]), pixels statistical analysis (e.g., co-occurrence matrices [9], Feature-based Interaction Map [10]) and complexity analysis (e.g., Fractal Dimension [1112]).

Recently, we have proposed a novel approach of texture analysis that achieved better results than most of the state-of-art methods 11314. It considers independent walkers leaving from each pixel of an image. With a given memory, each walker moves to one of its neighboring pixels according to the difference of intensity between these pixels, avoiding returning to recent visited pixels. Each generated trajectory, after a transient time $t$, ends in an attractor, i.e., a cycle of $p$ pixels from where the walker cannot escape. These transient times and attractors contain characteristics of the pixel organization in that image.

For instance, consider a partially self-avoiding deterministic walk, where a walker wishes to visit $N$ sites randomly distributed in a map of $d$ dimension. The walker can move from one to another site following the rule of, at each discreet time step, to go to the nearest site not visited in the previous $\mu$ steps. The agent performs a partially self-avoiding walk, where the self-avoidance is limited to the memory window $\tau=\mu-1$. The walker's behavior depends strictly on the data set configuration and on the starting site 1516. The walker's movements are entirely performed based on a neighborhood table, so that the distances among the sites are simply a way of ranking their neighbors. This feature leads to an invariance in scale transformations [17]. Each trajectory has an initial transient part of length $t$ and ends in a cycle with period $p$. Both the transient time and cycle period can be combined in the joint probability distribution $S_{\mu, d}^{(N)}(t, p)$. The simplest case to deal with the deterministic walker is to consider $\mu=0$, where the walker remains forever at the initial site $S_{0, d}^{(N)}(t, p)=\delta_{t, 0} \delta_{p, 1}$, where $\delta_{i, j}$ is the Kronecker's delta. Despite its triviality, this becomes interesting because it is the simplest situation of a stochastic walk [18. For a memoryless walker $(\mu=1)$, the walker, at each time step, the walker must leave the current site and go to the nearest one. After a very short transient time, the walker becomes trapped by a couple of mutually nearest neighbors. The transient time and period joint probability distribution, for $N \gg 1$, is [19]: $S_{1, d}^{(\infty)}(t, p)=\left[\Gamma\left(1+I_{d}^{-1}\right)\left(t+I_{d}^{-1}\right) / \Gamma\left(t+p+I_{d}^{-1}\right)\right] \delta_{p, 2}$, where $\Gamma(z)$ is the gamma function and $I_{d}$ is the normalized incomplete beta function $I_{d}=I_{1 / 4}[1 / 2,(d+1) / 2]$. In the limit $d \rightarrow \infty$, one is able to calculate it analytically [20]: $S_{2, \infty}^{(N)}(t, p)=e^{-[3 N(t+p-2)(t+p-3) / 2]} /\left[\left(3-\delta_{t, 0}\right) N\right]$. Analytical calculations [21] were also performed for the stochastic walk. When greater values 
of $\mu$ are considered, the cycle distribution is no longer peaked at $p_{\min }=\mu+1$, but presents a whole spectrum of cycles with period $p \geq p_{\min }$ [1516120].

The presentation is organized as follows. In Sec. 2, we review how this walk can be used to analyse and classify textures in images [1] and the $(t, p)$-joint distributions are used to generate a single signature vector for colored images. In Sec. 3, experiments are described and performed on standard colored texture images. These experiment show that the presented algorithm outperform traditional methods (Chromatic moments 22], Color differential [23] and to the gray-scale deterministic walker previous version [1]). We address our final remarks in Sec. 4,

\section{Walks on Color Images and Texture Signature}

In Ref. 1], the partially self-avoinding deterministic walk has been proposed as a tools to texture analysis and classification. This algorithm has been developed to analyze gray level texture. Here, we generalize the algorithm to be capable to deal with colored images. A colored image, of $N=M_{x} \times M_{y}$ pixels, is formed by three layers, each one representing a color (red, green and blue). In each layer the color intensity varies, in integer values, from 0 to 255 . Basically, the algorithm is exact the same of the previous version, but now one has a walker for each layer. The partial information of each walker is then joined in a single signature. Two pixels, $\left(x_{i}, y_{i}\right)$ and $\left(x_{j}, y_{j}\right)$ are considered neighbors if the Hamming distance is $d(i, j) \leq 2$.

A walker can only move according to the following rule: move to the nearest or furthest neighbor (i.e., the one which differs in the minimum or maximum intensity value, respectively, from the current position) and that has not been visited in the last $\mu(\mu \in[1, N])$ previous steps. This rule produces partially self-avoiding walks[1].

For each initial condition (i.e., the starting pixel), the walker produces a different trajectory. Notice, however, that different initial conditions can lead to the same attractor. Considering all pixels in the image as starting points, we compute the joint probability distribution of transient time $t$ and attractor period $p$, $S_{\mu, 2}^{(N)}(t, p)$. From the study of these distributions, using statistical techniques, it is possible to achieve a signature able to discriminate the image texture [1131417].

Previous studies have shown the $S_{\mu, 2}^{(N)}(t, p)$ potential application in the classification of gray-scale textures [11314]. Any change on image context affects the walk and, as a consequence, its joint probability distribution, achieved for an specific memory $\mu$ and walking rule. It makes this distribution a useful tool for texture analysis.

Many approaches can be used to extract relevant information from the joint probability distribution. Good results have been reported by the use of the histogram $h_{\tau}(n)$ on gray-scale textures analysis. This histogram represents the frequency that a trajectory of length $n$, where $n=t+p \geq \tau+2$, is performed by the walker, using a specific memory range $\tau$. From this histogram, a texture 
signature is easily built by selecting a total of $m$ descriptors. In the case of color textures, each channel is processed by the deterministic walk independently, thus resulting in a joint probability distribution and, as a consequence, a histogram specific for that channel.

$$
\boldsymbol{v}_{\tau}^{(C)}(m)=\left[h_{\tau}^{(C)}(\tau+2), h_{\mu}^{(C)}(\tau+3), \ldots, h_{\mu}^{(C)}(\tau+1+m)\right],
$$

where $C$ represent the channel explored in the texture. A texture signature which explores all color channels ( $\mathrm{R}, \mathrm{G}$ and $\mathrm{B})$ with memory $\mu$ is defined as:

$$
\boldsymbol{\psi}_{\tau}(m)=\left[\boldsymbol{v}_{\tau}^{(R)}(m), \boldsymbol{v}_{\tau}^{(G)}(m), \boldsymbol{v}_{\tau}^{(B)}(m)\right] .
$$

As the joint probability distribution is modified according to the $\mu$ value, it is also interesting to evaluate a texture pattern considering the signatures computed for different memories $\mu$ :

$$
\boldsymbol{\varphi}_{M}(m)=\left[\boldsymbol{\psi}_{0}(m), \boldsymbol{\psi}_{1}(m), \ldots, \boldsymbol{\psi}_{M}(m)\right] .
$$

\section{Experiments and Results}

Experiments have been performed to determine the configuration of the signature, in terms of memory and walk rule, that leads to the best color texture classification. Signatures were evaluated in a texture classification scheme. The VisTex 24] is a set of colored images widely used as benchmark for texture analysis (see Figure 1). This database has been used 640 samples of 40 classes. Each class contains 16 texture samples of $128 \times 128$ pixels.
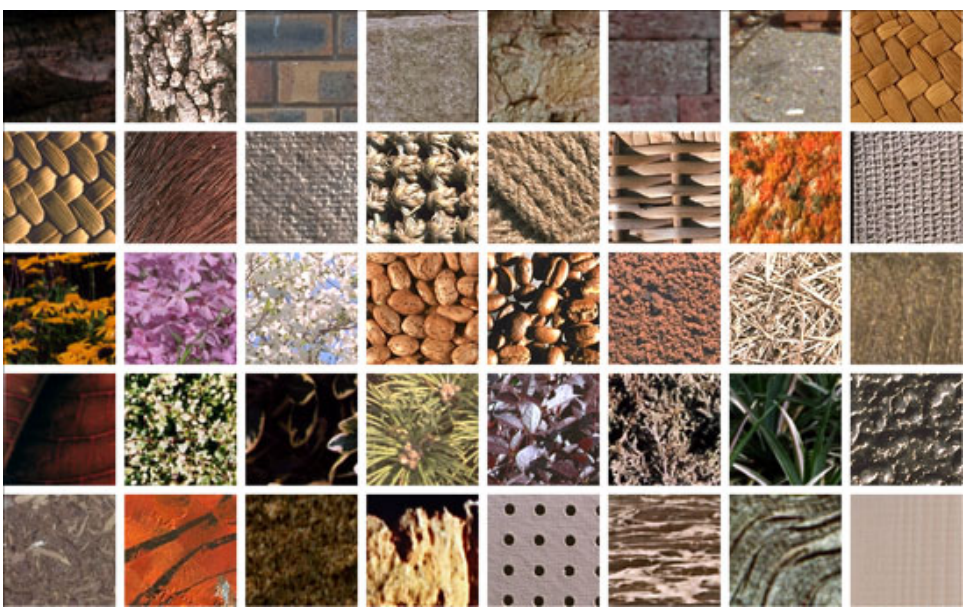

Fig. 1. One example of each of the 40 texture classes considered in the VisTex database

The evaluation of the signatures was performed using Linear Discriminant Analysis (LDA), a classification method based on supervisioned learning. LDA 
aims to find a linear combination of the descriptors (independent variables) that results in its class (dependent variable). This linear combination results in the data projection in a linear subspace where inter-classes variance is larger than intra-classes variance [2526]. The leave-one-out cross-validation strategy was also used, where each sample from the database is used for validation while remaining samples are used for training. This is repeated until all samples were used for validation.

Previous experiments [13] conducted over $S_{\mu, 2}^{(N)}(t, p)$ have shown that most image information is concentrated within few elements, where $0 \leq t \leq 4$ and $(\tau+2) \leq p \leq(\tau+5)$. From now on, we extract $m=4$ descriptors from $h_{\tau}(n)$ to compose the feature vectors $\boldsymbol{\psi}_{\tau}(m)$ (Eq. 2).

In Table 11, we show the success rate signatures (Eq. 21), evaluated according to different $\tau$ values, for both walking rules: agents guided to minimum and maximum intensity difference. The main difference between these walking rules concerns the regions where the attractors are found. The walk guided to the direction of the maximum pixel intensity difference locates attractors where modifications in image context are more abrupt, i.e., heterogeneous regions of the texture. Otherwise, walkers guided to the minimum intensity difference locate attractors, where the image present more homogeneous patterns. Heterogeneous regions are usually related to the presence of image contours or changes in texture patterns, both important visual attributes in the characterization of objects 2728. This explains the superior performance of the maximum difference in comparison to the minimum difference in the texture classification. As these walking rules produce signatures with different characteristics of the image, it would be interesting to evaluate the combination of these signatures into one (described as $\operatorname{Min} \cup \operatorname{Max}$ ). As expected, the union of heterogeneous and homogeneous texture information provides a more powerful tool for image analysis. We also note that, independent of the used walking rule, the success rate decreases as the memory increases. Each walker produces a self-avoiding trajectory, which depends on the memory used. This memory avoids that the walker visits some pixels of the image, so that, a better exploration of the image is performed. As the memory increases, more the agent has to walk to find an attractor. Higher memories endangers the local exploration of the texture by the walker, which reflects on the decreases of the success rate.

Table 2 shows the results obtained when multiple memories $\tau$ are used to compose the texture signature $\boldsymbol{\varphi}_{M}(4)(\mathrm{Eq} .3$. Note that this signature provides

Table 1. Success rate for $\boldsymbol{\psi}_{\tau}(4)$ signature (Eq. 2) using different $\tau$ values and walking rules in the VisTex database

\begin{tabular}{|c|c|c|c|c|c|c|}
\hline & \multicolumn{6}{|c|}{ Memory( $(\tau)$} \\
Texture signature & 0 & 1 & 2 & 3 & 4 & 5 \\
\hline Min & $56.87 \%$ & $44.37 \%$ & $33.91 \%$ & $27.06 \%$ & $20.47 \%$ & $15.14 \%$ \\
Max & $90.16 \%$ & $65.78 \%$ & $76.09 \%$ & $59.84 \%$ & $60.31 \%$ & $30.16 \%$ \\
Min $\cup$ Max & $94.37 \%$ & $81.72 \%$ & $81.41 \%$ & $74.06 \%$ & $70.31 \%$ & $50.31 \%$ \\
\hline
\end{tabular}


Table 2. Success rate for the texture signature $\varphi_{M}$ (4) (Eq. 3) combining different $\tau$ values in the VisTex database

\begin{tabular}{|c|c|c|c|c|c|}
\hline & \multicolumn{5}{|c|}{ Multiple Memories $(M)$} \\
Texture signature & 1 & 2 & 3 & 4 & 5 \\
\hline Min & $74.21 \%$ & $77.65 \%$ & $78.75 \%$ & $79.06 \%$ & $80.94 \%$ \\
Max & $93.90 \%$ & $94.53 \%$ & $94.69 \%$ & $94.53 \%$ & $95.00 \%$ \\
Min $\cup$ Max & $96.25 \%$ & $96.09 \%$ & $97.03 \%$ & $96.87 \%$ & $96.72 \%$ \\
\hline
\end{tabular}

an increase in success rates in comparison to the results of Table 1, where each memory was independently evaluated. This approach enable us to characterize a texture using information collected from different scales, thus providing a more efficient image classification.

Finally, we compare our algorithm to other texture analysis methods, which are briefly described below. Chromaticity Moments: it is based on the concept of chromaticities as defined within the CIE XYZ color space, where each image pixel results in a pair of $(x, y)$ chromaticity values. From the chromaticity values distribution, moments are computed to compose a feature vector that allows to characterize the image in terms of color and texture 22. Color Differential: the method uses a fractal measure on the interaction between color bands of the image. It is based on the study of the intercolor volume enclosed between each two color surfaces. The method also considers the CIE-chromaticity value for material color information as an additional feature of the image [23]. This method is originally proposed for the segmentation of digital images, but it can be easily adapted to solve problems of texture classification.

Table 3. Comparison of the success rates for different color texture methods in the VisTex database

\begin{tabular}{|c|c|c|}
\hline Method & Images correctly classified & Success rate(\%) \\
\hline Chromaticy Moments & 534 & 83.44 \\
\hline Color Differential & 599 & 93.59 \\
\hline Deterministic Walk (color) & 621 & 97.03 \\
\hline Deterministic Walk (gray) & 597 & 93.28 \\
\hline
\end{tabular}

In Table 3 we show the success rate of our method to Chromaticity Moments and the Color Differential methods. In this comparison, the best result has been achieved by our method (using Eq. 3 with $M=3$, for minimum and maximum walk rules). We also consider in this comparison the results of our method when disregarding the color information of the texture, i.e., walks performed only over the gray-scale version of the texture, where one clearly sees that a colored image has more information than a single gray-scale image.

We note that the deterministic walker's best result achieved better results from compared color texture methods. In fact, the use of $\boldsymbol{\psi}_{\mu}$ signature computed for $\mu=0$, and both minimum and maximum directions, as described in Table 1 . provides a result better than Chromaticy Moments and Color Differential, thus 
evidencing the quality of the proposed approach in color texture analysis. Results also show that the use of color information provides a better identification of texture patterns, as the deterministic walker's performance over color texture is superior in comparison to gray-scale textures.

\section{Conclusion}

We have presented a generalized version of the texture feature extraction based on partially self-avoiding deterministic walks to deal with color textures. The proposed method uses walkers that explore the different color channels of an image on a given scale (memory). A simple signature vector is computed and numerical experiments have been conducted with a color texture database to evaluate the success rates. Our method has been confronted with the previous one and with other two state-of-art color texture algorithms. It has overcame all of them. The result demonstrates the great potential of the proposed method, combining color information of the texture and be capable of recognize it.

\section{Ackowledgements}

A.R.B. acknowledges support from FAPESP (2006/54367-9). A.S.M. acknowledges support from CNPq (303990/2007-4 and 476862/2007-8). O.M.B. acknowledges support from CNPq (306628/2007-4 and 484474/2007-3) and FAPESP (2006/54367-9).

\section{References}

1. Backes, A.R., Gonçalves, W.N., Martinez, A.S., Bruno, O.M.: Texture analysis and classification using deterministic tourist walk. Pattern Recognition 43, 685-694 (2009)

2. Tuceryan, M., Jain, A.K.: Texture analysis. In: Handbook of Pattern Recognition and Computer Vision, pp. 235-276 (1993)

3. Wu, C.M., Chen, Y.C., Hsieh, K.S.: Texture features for classification of ultrasonic liver images. IEEE Transactions on Medical Imaging 11, 141-152 (1992)

4. Heidelbach, F., Kunze, K., Wenk, H.R.: Texture analysis of a recrystallized quartzite using electron diffraction in the scanning electron microscope. Journal of Structural Geology, 91-104 (2000)

5. Anguiano, E., Oliva, A.I., Aguilar, M.: Surface texture parameters as a tool to measure image quality in scanning probe microscope. Ultramicroscopy $77(3)$, 195-205 (1999)

6. Zhang, J., Tan, T.: Brief review of invariant texture analysis methods. Pattern Recognition 35(3), 735-747 (2002), http://lear.inrialpes.fr/pubs/2002/ZT02

7. Azencott, R., Wang, J.-P., Younes, L.: Texture classification using windowed Fourier filters. IEEE Trans. Pattern Anal. Mach. Intell. 19(2), 148-153 (1997)

8. Jain, A.K., Farrokhnia, F.: Unsupervised texture segmentation using gabor filters. Pattern Recogn. 24(12), 1167-1186 (1991) 
9. Haralick, R.M.: Statistical and structural approaches to texture. Proc. IEEE 67(5), 768-804 (1979)

10. Chetverikov, D.: Texture analysis using feature-based pairwise interaction maps. Pattern Recognition 32(3), 487-502 (1999)

11. Chaudhuri, B.B., Sarkar, N.: Texture segmentation using fractal dimension. IEEE Trans. Pattern Anal. Mach. Intell. 17(1), 72-77 (1995)

12. Backes, A.R., Bruno, O.M.: A new approach to estimate fractal dimension of texture images. In: Elmoataz, A., Lezoray, O., Nouboud, F., Mammass, D. (eds.) ICISP 2008. LNCS, vol. 5099, pp. 136-143. Springer, Heidelberg (2008)

13. Campiteli, M.G., Martinez, A.S., Bruno, O.M.: An image analysis methodology based on deterministic tourist walks. In: Sichman, J.S., Coelho, H., Rezende, S.O. (eds.) IBERAMIA 2006 and SBIA 2006. LNCS (LNAI), vol. 4140, pp. 159-167. Springer, Heidelberg (2006)

14. Backes, A.R., Bruno, O.M., Campiteli, M.G., Martinez, A.S.: Deterministic tourist walks as an image analysis methodology based. In: Martínez-Trinidad, J.F., Carrasco Ochoa, J.A., Kittler, J. (eds.) CIARP 2006. LNCS, vol. 4225, pp. 784-793. Springer, Heidelberg (2006)

15. Lima, G.F., Martinez, A.S., Kinouchi, O.: Deterministic walks in random media. Phys. Rev. Lett. 87(1), 010603 (2001)

16. Stanley, H.E., Buldyrev, S.V.: Statistical physics - the salesman and the tourist. Nature (London) 413(6854), 373-374 (2001)

17. Campiteli, M.G., Batista, P.D., Kinouchi, O., Martinez, A.S.: Deterministic walks as an algorithm of pattern recognition. Physical Review E (Statistical, Nonlinear, and Soft Matter Physics) 74(2), 026703 (2006)

18. Martinez, A.S., Kinouchi, O., Risau-Gusman, S.: Exploratory behavior, trap models, and glass transitions. Phys. Rev. E 69(1), 017101 (2004)

19. Terçariol, C.A., Martinez, A.S.: Analytical results for the statistical distribution related to a memoryless deterministic walk: dimensionality effect and mean-field models. Phys Rev. E 72

20. Tercariol, C.A.S., Martinez, A.S.: Influence of memory in deterministic walks in random media: Analytical calculation within a mean-field approximation. Phys. Rev. E 78(3), 031111 (2008)

21. Risau-Gusman, S., Martinez, A.S., Kinouchi, O.: Escaping from cycles through a glass transition. Phys. Rev. E 68, 016104 (2003)

22. Paschos, G.: Fast color texture recognition using chromaticity moments. Pattern Recognition Letters 21(9), 837-841 (2000)

23. She, A.C., Huang, T.S.: Segmentation of road scenes using color and fractal-based texture classification. In: ICIP, vol. (3), pp. 1026-1030 (1994)

24. Vision texture database (2009), http://vismod.media.mit.edu/vismod/imagery/VisionTexture/vistex.html

25. Everitt, B.S., Dunn, G.: Applied Multivariate Analysis, 2nd edn. Arnold, London (2001)

26. Fukunaga, K.: Introduction to Statistical Pattern Recognition, 2nd edn. Academic Press, London (1990)

27. Loncaric, S.: A survey of shape analysis techniques. Pattern Recognition 31(9), 983-1001 (1998)

28. da Fontoura Costa, L., Cesar Jr., R.M.: Shape Analysis and Classification: Theory and Practice. CRC Press, Boca Raton (2000) 